\title{
Estrategias de tratamiento antibiótico para la neumonía adquirida en la comunidad en adultos
}

\section{Antibiotic treatment strategies for community- acquired pneumonia in adults}

Natalia Andrea Rendón García, MD. (1), JaVier Garzón, MD. ${ }^{(2)}$

${ }^{(1)}$ Residente de Primer año de Medicina Interna, Departamento de Medicina Interna, Pontificia Universidad Javeriana, Hospital Universitario San Ignacio, Bogotá, Colombia.

(2)Internista, Infectólogo, Unidad de Infectología, Departamento de Medicina Interna, Hospital Universitario San Ignacio, Pontificia Universidad Javeriana, Bogotá, Colombia.

Correspondencia: Javier Garzón. Correo electrónico: garzonjavier@yahoo.com Recibido: 8/12/14, Aceptado: 10/12/14.

\begin{abstract}
Referencia
Postma Douwe, Van Werkhoven Cornelis, Van Elden Leontine, Thijsen Steven, Hoepelman Andy, Kluytmans Jan, et al; CAP-START Study Group. Antibiotic treatment strategies for community-adquired pneumonie in adults. N Engl J Med. 2015; 372(14):1312-23.
\end{abstract}

\section{Pregunta}

En pacientes con sospecha clínica de neumonía adquirida en la comunidad, que no requieren manejo en la unidad de cuidados intensivos ¿es la monoterapia con betalactámicos no inferior a la terapia dual de betalactámicos y macrólidos y a la monoterapia con fluoroquinolonas, con relación a la mortalidad por cualquier causa a los 90 días?

\section{Métodos}

\section{Diseño}

Ensayo clínico de no inferioridad pragmático, cruzado, de asignación aleatoria por conglomerados. Margen de no inferioridad calculado de $3 \%$.

\section{Cegamiento}

Aleatorización de la secuencia de tratamiento que recibiría cada hospital participante.

\section{Periodo de seguimiento}

Seguimiento llevado a cabo en dos años, en periodos definidos por cuatro meses para cada esquema de tratamiento.

\section{Lugar}

Siete hospitales holandeses, dos de los cuales eran universitarios.

\section{Pacientes}

3.325 participante de 18 años o más, con sospecha clínica de neumonía adquirida en la comunidad que requirieron manejo intrahospitalario en salas diferentes a la unidad de cuidado intensivo. Se excluyeron aquellos con fibrosis quística.

\section{Intervención}

Monoterapia con betalactámi$\cos (n=993)$, terapia combinada con betalactámicos mas macrólido $(\mathrm{n}=1.055)$, monoterapia con fluoroquinolona $(n=1.277)$. Durante periodos de cuatro meses 
Tabla 1. Resultados de la comparación entre las distintas estrategias de antibióticos.

\begin{tabular}{|lcc|}
\hline \multicolumn{1}{|c}{ Desenlace } & Blact vs Blactam + mac & Blact vs FQ \\
Riesgo ajustado de muerte a 90 días. & 1,9 (IC 90\% 0,6-4,4) & $-0,6$ (IC 90\% 2,8-1,9) \\
$\begin{array}{l}\text { Razón de tasas ajustada para alta vivo en la } \\
\text { población de intención a tratar }\end{array}$ & 0,87 (IC 95\% 0,78-0,97) & 1,04 (IC 95\% 0,94-1,16) \\
$\begin{array}{l}\text { Razón de tasas ajustada para inicio de } \\
\text { antibiótico oral en la población de intención } \\
\text { a tratar }\end{array}$ & 0,97 (IC 95\% 0,86-1,09) & 1,29 (IC 1,15-1,46) \\
$\begin{array}{l}\text { Odds ratio de las complicaciones en la } \\
\text { población de intención a tratar }\end{array}$ & 1,06 (IC 95\% 0,76-1,48) & 1,02 (IC 95\% 0,73-1,41) \\
\hline
\end{tabular}

Blact: Betalactámico, mac: macrólido, FQ: fluoroquinolona

consecutivos, la monoterapia con betalactámicos, la terapia dual con betalactámicos más macrólidos y la monoterapia con fluoroquinolonas se usaron como tratamiento empírico para los pacientes seleccionados. Estos fueron tratados y valorados de acuerdo con la estrategia terapéutica que fue asignada en la fecha de admisión. Se informaba a los médicos tratantes acerca de la estrategia antibiótica utilizada, la cual se basaba en las guías holandesas de 2005. Al finalizar el periodo correspondiente (cuatro meses), se cambiaba el esquema terapéutico, según el orden dado por la aleatorización inicial, hasta cumplir con un periodo de dos años.

\section{Desenlace primario}

Mortalidad a los 90 días por cualquier causa después de la admisión.

\section{Otros desenlaces}

- Tiempo de inicio del tratamiento oral.

- Estancia hospitalaria.

- Complicaciones menores y mayores durante la estancia hospitalaria.

\section{Seguimiento de pacientes}

Se analizó mortalidad a 90 días por intención a tratar.

\section{Resultados}

Los grupos poblacionales en estudio no tuvieron mayores diferencias en cuanto a sus características demográficas. La edad promedio de los pacientes fue 70 años, la mayoría de género masculino, que presentaron tres días de síntomas. Las condiciones preexistentes que se describieron fueron enfermedad cardiovascular, enfermedad pulmonar obstructiva crónica o asma, diabetes mellitus, cáncer, enfermedad renal crónica o síndrome nefrótico y VIH/SIDA. En cuanto a las escalas de severidad, el PSI (del inglés Pneumonia Severity Index) se calculó en promedio en un puntaje de 84, y el CURB-65 fue de 1 en los tres grupos de estudio.

La diferencia absoluta en el riesgo ajustado de muerte entre la estrategia de betalactámicos y la estrategia dual entre betalactámico más macrólido, fue 1,9 (IC 90\%, 0,6-4,4) en favor de la estrategia con betalactámico mientras que la diferencia absoluta entre la estrategia de betalactámico y la monoterapia con fluoroquinolona fue $-0,6$ (IC $90 \%, 2,8-1,9)$. Estos intervalos de confianza no incluyeron el margen pre especificado del 3\%, con lo que se demostró la no inferioridad de la estrategia de monoterapia con betalactámico a la estrategia dual de betalactámico más macrólido y a la monoterapia con fluoroquinolonas.

En la adherencia a la estrategia de manejo antibiótico y al antibiótico, las diferencias de riesgo 
absoluto ajustado fueron similares. Los estimativos se obtuvieron en el análisis de sensibilidad de pacientes con neumonía adquirida en la comunidad confirmada radiológicamente y un análisis de mortalidad a los 30 días.

\section{Conclusión}

En quienes se sospechó neumonía adquirida en la comunidad y fueron admitidos en el hospital en unidades diferentes a la unidad de cuidado intensivo, la monoterapia con betalactámicos como tratamiento empírico demostró ser no inferior a la terapia dual de betalactámicos más macrólido o la monoterapia con fluoroquinolona en términos de mortalidad a 90 días por cualquier causa.

El promedio de estancia hospitalaria fue de seis días para todas las estrategias, pero el rango intercuartílico más alto se documentó en el grupo de betalactámico más macrólido. La duración promedio del tratamiento venoso fue de tres días en la estrategia de la monoterapia con fluoroquinolona y de cuatro días durante las demás estrategias de tratamiento. La proporción de pacientes que iniciaron antibióticos orales fue del $27 \%$ durante la terapia con fluoroquinolonas, en comparación con un 13 y un $10 \%$ durante el periodo de manejo con monoterapia con betalactámicos y la terapia dual, respectivamente. No hubo diferencias significativas en la incidencia de complicaciones mayores o menores entre las tres estrategias.

\section{Comentario}

El artículo describe que la monoterapia con betalactámicos es no inferior a la terapia dual de betalactámico más macrólido y a la monoterapia con fluoroquinolonas, en cuanto a mortalidad a 90 días por cualquier causa, en una población holandesa. Para la apreciación objetiva de dicha conclusión, es importante tener en cuenta las revisiones previas reportadas en la literatura respecto a este tópico.

Las guías internacionales, tanto las norteamericanas como las inglesas, sugieren como manejo antibiótico empírico, en los pacientes adultos con neumonía adquirida en la comunidad, un esquema dual de tratamiento con betalactámicos y macrólidos (1), en vista de que esta población puede estar infectada por gérmenes atípicos (2), si bien la incidencia de los mismos varía según diferentes series.

La literatura actual cuenta con información contradictoria sobre el uso de macrólidos como terapia combinada en pacientes con neumonía. Robert et al. (3) documentaron las limitaciones metodológicas de los estudios, para realizar la interpretación del beneficio real de la terapia combinada con macrólidos en esta población, en especial, ante la ausencia de una prueba microbiológica diagnóstica que permitiera la confirmación de infección por gérmenes atípicos. Con base en lo anterior, las recomendaciones sobre el uso de macrólidos en terapia empírica combinada, se dan con base en la frecuencia de infección por dichos gérmenes en el paciente con neumonía adquirida en la comunidad, en vista de la imposibilidad de acceso a una herramienta para el diagnóstico confirmatorio de infección por atípicos (3).

Existen varios metaanálisis, entre ellos el de Nie et al. (4), en el que se analiza la terapia dual frente a la monoterapia con betalactámicos, en términos de mortalidad. Consiste en una revisión sistemática, donde la mayoría de los estudios son observacionales, y se concluye que la terapia combinada tiene un beneficio en la sobrevida por encima de la monoterapia con betalactámicos. Sin embargo, en la discusión de esta publicación se hace referencia a las posibles explicaciones de este desenlace, y se atribuye el beneficio de los macrólidos a una acción antiinflamatoria, tal como se ha discutido en la EPOC (4).

Es importante aclarar sin embargo, que también se menciona la población más joven y con menos comorbilidades, que recibió manejo con macrólidos y que posiblemente impacte favorablemente en la mortalidad hacia uno de los grupos de estudio debido a su mayor sobrevida. Por otra parte, una revisión de Cochrane del año 2012 acerca de 
ensayos clínicos aleatorios, donde se analizó mortalidad y falla terapéutica sobre el cubrimiento antibiótico empírico de patógenos atípicos, concluyó que no se evidencia beneficio en la sobrevida o eficacia clínica con el cubrimiento empírico para atípicos en pacientes hospitalizados por neumonía adquirida en la comunidad (5). Asociado a este resultado, se resalta la evidencia que favorece la resistencia a los macrólidos y el riesgo cardiovascular asociado al mismo $(6,7)$.

Así pues, la evidencia en cuanto al tratamiento empírico dual con macrólidos, es contradictoria, si se tiene en cuenta no sólo el déficit en la metodología de los estudios realizados, sino las diferencias en la prevalencia de la infección de gérmenes atípicos en distintas regiones del mundo, lo que hace imperativo el desarrollo de estudios clínicos que deben analizarse a la luz de la población estudiada.

En este estudio llama la atención la baja incidencia de infección por atípicos, dato que no se aplica a la población colombiana, en quienes la infección por estos gérmenes puede alcanzar cifras hasta de un $20 \%$, aun a pesar de la dificultad que representa la identificación microbiológica de estos gérmenes ante la ausencia de una prueba diagnóstica rápida y confiable. Además, se recalca el puntaje de CURB-65 obtenido en la población de estudio, pues por el promedio de edad, los pacientes ya clasificarían en este grupo, hecho que limita, en cierta forma, la aplicabilidad de las conclusiones finales del artículo a nuestra población.

\section{Conflictos de intereses}

Los autores no declaran algún conflicto de interés.

\section{Bibliografía}

1. Garin N, Genné D, Carballo S, Chuard C, Eich G, Hugli O, et al. $\beta$-Lactam monotherapy vs. $\beta$-lactam-macrolide combination treatment in moderately severe community-acquired pneumonia: a randomized noninferiority trial. JAMA Int Med. 2014;174(12).

2. Cillóniz C, Ewig S, Polverino E, Marcos MA, Esquinas C, Gabarrús A. Microbial aetiology of community-acquired pneumonia and its relation to severity. Thorax. 2011;66(4):340-6.

3. File TM Jr, Marrie TJ. Does empiric therapy for atypical pathogens improve outcomes for patients with CAP? Infect Dis Clin North Am. 2013;27(1):99-104-14.

4. Nie W, Li B, Xiu Q. $\beta$-Lactam/macrolide dual therapy versus $\beta$-Lactam monotherapy for the treatment of community- adquired pneumoniae in adults: a systematic review and metaanalysis. J Antimicrob Chemother. 2014;69(6):1441-6.

5. Eliakim-Raz N, Robenshtok E, Shefet D, Gafter-Gvili A, Vidal L, Paul M, et al. Empiric antibiotic coverage of atypical pathogens for community-adquired pneumonie in hospitalized adults. Cochrane Datebase Syst Rev. 2012;12:9.

6. Vanderkooi OG, Low DE, Green K, Powis JE, McGeer A. Predicting antimicrobial resistance in invasive pneumococcal infections. Clin Infect Dis. 2005; 40(9):1288-97.

7. Ray WA, Murray KT, Hall K, Arbogast PG, Stein CM. Azithromycin and the risk of cardiovascular death. N Engl J Med. 2012;366(20):1881-90. 